\title{
Exponential Formulas and Lie Algebra Type Star Products
}

\author{
Stjepan MELJANAC ${ }^{\dagger}$, Zoran $\check{S} K O D A^{\dagger}$ and Dragutin SVRTAN ${ }^{\ddagger}$ \\ $\dagger$ Division for Theoretical Physics, Institute Rudjer Bošković, \\ Bijenička 54, P.O. Box 180, HR-10002 Zagreb, Croatia \\ E-mail:meljanac@irb.hr, zskoda@irb.hr \\ $\ddagger$ Department of Mathematics, Faculty of Natural Sciences and Mathematics, \\ University of Zagreb, HR-10000 Zagreb, Croatia \\ E-mail:dsurtan@math.hr
}

Received May 26, 2011, in final form March 01, 2012; Published online March 22, 2012

http://dx.doi.org/10.3842/SIGMA.2012.013

\begin{abstract}
Given formal differential operators $F_{i}$ on polynomial algebra in several variables $x_{1}, \ldots, x_{n}$, we discuss finding expressions $K_{l}$ determined by the equation $\exp \left(\sum_{i} x_{i} F_{i}\right)\left(\exp \left(\sum_{j} q_{j} x_{j}\right)\right)=\exp \left(\sum_{l} K_{l} x_{l}\right)$ and their applications. The expressions for $K_{l}$ are related to the coproducts for deformed momenta for the noncommutative space-times of Lie algebra type and also appear in the computations with a class of star products. We find combinatorial recursions and derive formal differential equations for finding $K_{l}$. We elaborate an example for a Lie algebra $s u(2)$, related to a quantum gravity application from the literature.
\end{abstract}

Key words: star product; exponential expression; formal differential operator

2010 Mathematics Subject Classification: 81R60; 16S30; 16S32; 16A58

\section{Introduction}

Deformation quantization $[2,3,6,7,15,16]$ studies the associative algebra deformations of the algebra of (usually smooth) functions on a Poisson manifold, with a prescription that a linear part is proportional to the Poisson structure. Thus the deformed algebra is, for every specialization of the deformation parameter, isomorphic as a vector space to the undeformed. Some deformations however can appear with a different motivation, namely as algebras of functions on a space or spacetime $[1,9,10,12,14]$ underlying a noncommutative field theory (motivated by Planckscale physics); in that case the Poisson structure viewpoint to deformation is not of primary concern, but rather various additional structures and symmetries enabling to establish elements of geometry and field theory on such a spacetime, e.g. the kappa-spacetime [1, 17, 18, 19]. Both classical and quantum field theories can be studied on such noncommutative spacetimes. The deformation relation to the commutative spacetime, for such applications, is important for the correct limit of physics at the ordinary scales, and as a method to define various procedures by help of realizations by commutative coordinates (and some additional operators on commutative functions). In the deformation quantization the question of existence and uniqueness of a star product (possibly with additional symmetry constraints), for a given Poisson algebra is one of the central questions. On the other hand, in the study of noncommutative space-time, the noncommutative base algebra is a given at start and exploring a distinguished isomorphism to the underlying vector space some commutative algebra is just a method. Thus our perspective and questions are rather different from the deformation quantization, though some formulas and concepts make sense in either context. 
Technical tools to introduce geometrical notions and calculus needed to do field theory on noncommutative spaces is in progress. Even for simple cases, e.g. the case of linear Poisson structures, the choice of a star product within an equivalence class, can make definitions of additional structures more or less accessible.

There is often the case that the generators of a noncommutative algebra can be realized in terms of differential operators (elements in a Weyl algebra); sometimes one allows also formal expressions in differential operators up to infinite order. Such realizations are very useful in physics computations. Structures of our concern include coproducts, deformed derivatives and exterior calculi $[4,18,19,22]$ and methods include realizations of noncommutative algebras via series in formal differential operators. We have been using systematically such realizations in recent works (see e.g. [11, 17, 18, 19]). These realizations are also used to treat the star products, as often an action by noncommutative variables in differential operators on the Fock space gives the required isomorphism of vector spaces with a space of commutative expressions. In general, the star product does not extend from polynomials to the formal power series, but we can include some subspace of formal power series; almost all formalisms include at least the exponential series, possibly of a polynomial argument. In particular, Fourier-type expansions of noncommutative functions into noncommutative deformations of plane waves, are often used $[1,12,13,14]$ and many formulas are proved in practice for bases formed by such formal exponential expressions. Thus we here study some aspects of an abstract version of the typical realizations of such exponentials of formal differential operators. We exhibit some facts and algorithms concerning the exponential series of differential operators of a type often needed in this line of work and especially in the case when we deal with a noncommutative space-time of Lie algebra type $[1,9,10,19]$. Some of the mathematical statements here may be used more generally than for the purposes of noncommutative geometry.

Given a finite-dimensional Lie algebra $\mathfrak{g}$ over a field $\boldsymbol{k}$, one sometimes finds convenient to express the noncommutative product on the enveloping algebra $U(\mathfrak{g})$ by transfering it via a vector space isomorphism to a "Lie type star product" on the underlying space of the symmetric algebra $S(\mathfrak{g})$. As it is well known, both $U(\mathfrak{g})$ and $S(\mathfrak{g})$ have a unique Hopf algebra structure in which the elements $x$ in $\mathfrak{g}$ are primitive, i.e. $\Delta(x)=1 \otimes x+x \otimes 1$. For some purposes, e.g. for introducing the deformed derivatives [18], the coalgebra isomorphisms $\xi: S(\mathfrak{g}) \stackrel{\simeq}{\longrightarrow} U(\mathfrak{g})$ (linear isomorphisms satisfying $\Delta(\xi(x))=(\xi \otimes \xi)(\Delta(x))$ for all $x \in S(\mathfrak{g}))$, are better than other linear isomorphisms; we also naturally require that $\xi$ restrict to the identity on $\mathbf{k} \oplus \mathfrak{g} \subset S(\mathfrak{g})$. The star product on $S(\mathfrak{g})$ transported by coalgebra isomorphism $\xi$ and defined by $f * g=$ $\xi^{-1}(\xi(f) \cdot \xi(g))$, will then have a number of special features, including a well-defined coproduct on deformed derivatives (interpreted as the deformed momenta $[12,17,18,19]$ ) and interesting noncommutative differential calculi [22].

The coalgebra isomorphism $\xi$ may be replaced by equivalent data. Namely, let $\boldsymbol{k}$ be of characteristic zero, in a basis $\hat{x}_{1}, \ldots, \hat{x}_{n}$ of $\mathfrak{g}$ with structure constants $C_{i j}^{k} \in \boldsymbol{k}$ defined by $\left[\hat{x}_{i}, \hat{x}_{j}\right]=$ $C_{i j}^{k} \hat{x}_{k}$ (with Einstein summation convention); we view this basis also as a set of generators of the enveloping algebra $U(\mathfrak{g})$. Then, by a result of [18], there is 1-1 correspondence between coalgebra isomorphisms $\xi$ 's and matrices $\left(\phi_{j}^{i}\right)_{i, j=1, \ldots, n}$ of formal power series $\phi_{j}^{i}=\phi_{j}^{i}\left(\partial^{1}, \ldots, \partial^{n}\right)$ in $n$ dual variables $\partial^{1}, \ldots, \partial^{n} \in \mathfrak{g}^{*}$, such that $\phi_{j}^{i}$ 's satisfy a system of formal differential equations [18]

$$
\phi_{j}^{l} \frac{\partial}{\partial\left(\partial^{l}\right)}\left(\phi_{i}^{k}\right)-\phi_{i}^{l} \frac{\partial}{\partial\left(\partial^{l}\right)}\left(\phi_{j}^{k}\right)=C_{i j}^{s} \phi_{s}^{k}
$$

That system of equations for $\phi_{i}^{j}$ is, on the other hand, equivalent to the requirement that the "realization" $\hat{x}_{i} \mapsto \hat{x}_{i}^{\phi}=\sum_{j=1}^{n} x_{j} \phi_{i}^{j}$ extends to a homomorphism $(-)^{\phi}: u \mapsto u^{\phi}$ of associative algebras from $U(\mathfrak{g})$ into the semicompleted $n$-th Weyl algebra $A_{n, \mathbf{k}}$ (which is as a vector space identified to $S\left(\mathfrak{g} \otimes \hat{S}\left(\mathfrak{g}^{*}\right)\right.$; moreover as discussed in [18], as an algebra, it is a smash product 
$\left.S(\mathfrak{g}) \sharp \hat{S}\left(\mathfrak{g}^{*}\right)\right)$. Here $\hat{S}(V)$ denotes the completed symmetric algebra of the finite-dimensional vector space $V$ (isomorphic to the ring of formal power series in indeterminates which form a basis in $V)$.

While $\hat{x}_{1}, \ldots, \hat{x}_{n}$ do not commute, the corresponding commutative variables $x_{1}, \ldots, x_{n}$ will be the generators of $S(\mathfrak{g})$.

The map $\hat{x} \mapsto \hat{x}^{\phi}$ extends multiplicatively to a homomorphism from $U(\mathfrak{g})$ to the Weyl algebra $\hat{A}_{n, \mathbf{k}}$ semicompleted with respect to the order of differential operator (we allow series in partial derivatives but only polynomials in coordinates $\left.x_{1}, \ldots, x_{n}\right)$.

It has been shown in [18] that the inverse of $\xi$ is given by the composition of this map extending $\hat{x} \mapsto \hat{x}^{\phi}$ and action of the (semicompleted) Weyl algebra on the Fock vacuum $|0\rangle=1_{S(\mathfrak{g})}$.

Yet another datum equivalent to $\xi$ is a vector valued function $K=\left(K_{1}, \ldots, K_{n}\right)$ determined by the statement

$$
\exp \left(\sum_{i} k_{i} \hat{x}_{i}^{\phi}\right) \exp \left(\sum_{j} q_{j} x_{j}\right)=\exp \left(\sum_{l} K_{l}(k, q) x_{l}\right)
$$

where, on the left-hand side, we use the usual Fock action of $\hat{A}_{n, \boldsymbol{k}}$ on $S(\mathfrak{g})$, extended appropriately to the power series involved, and $k=\left(k_{1}, \ldots, k_{n}\right), q=\left(q_{1}, \ldots, q_{n}\right) \in \mathbf{k}^{n}$. If $\boldsymbol{k}=\mathbf{C}$ one may prefer to put $\sqrt{-1}$ in front of all exponentials in (1) and this introduces Fourier-like expressions (cf. Section 4). This article discusses two kinds of issues:

- general questions on formal operator formulas like (1) in formal setup.

- specifics of exponentials appearing in our setup, and finding function $K$. In particular, in Section 4, we discuss function $K$ for a couple of realizations for $\phi$ in the case of $s u(2)$.

The simplest case is, of course, $K(k, q)=k+q$ in which case $\xi=e$ is the coexponential (or symmetrization) map, given by

$$
e: y_{1} \cdots y_{l} \mapsto \frac{1}{l !} \sum_{\sigma \in \Sigma(l)} \hat{y}_{\sigma(1)} \cdots \hat{y}_{\sigma(l)}: \quad S(\mathfrak{g}) \rightarrow U(\mathfrak{g}),
$$

for all $l$, and any elements $y_{1}, \ldots, y_{l}$ in $\mathfrak{g} \subset S(\mathfrak{g})$, where $\hat{y}_{1}, \ldots, \hat{y}_{l}$ are the same elements as $y_{1}, \ldots, y_{l}$, but understood as the "noncommuting" elements in $\mathfrak{g} \in U(\mathfrak{g})$.

We should first point out that in general (exception: trivial case with $\mathfrak{g}$ Abelian) the star product given by $f * g=\xi^{-1}(\xi(f) \cdot \xi(g))$ can not be continuously and bilinearly extended to all of $\hat{S}(\mathfrak{g})$; namely one can indeed find bad power series $f$ for which $\hat{x}_{i} * f=\sum_{j} x_{j} \phi_{i}^{j}(f)$ can not be consistently written even as a formal power series (the coefficients of monomials in $\phi_{i}^{j}(f)$ diverge). In particular, for an arbitrary $\phi_{i}^{j}$ it may happen that the star product even among the exponentials of the form $\exp \left(\sum_{j} q_{j} x_{j}\right)$ is ill defined. To have some control of the issue, we establish some techniques of calculating star products of such exponentials, where one of the exponentials is $\exp \left(\lambda \sum_{j} k_{j} x_{j}\right)$ and $\lambda$ is a formal commuting variable. Then the question of convergence can be studied for the result of the calculation, after specializing our results from formal $\lambda$ to an actual value.

While we discuss existence of function $K=K(k, q)$ below in this article, let us suppose its existence now and discuss the coproduct; namely if we know $K$ we can determine the formula for coproduct (for examples see Section 4).

We can then introduce vector function $K_{0}^{-1}$ which is the inverse of

$$
K_{0}: k \mapsto K(k, 0) .
$$


The formula for the star product for exponentials must be a linear extension (whenever it converges) of the formula for the polynomials given by $\xi(f * g)=\xi(f) \cdot \xi(g)$. Here $\xi$ is the inverse of the map $U(\mathfrak{g}) \cong S(\mathfrak{g})$ given by $u \mapsto u^{\phi}|0\rangle$, i.e. on the monomials $\hat{x}_{j 1} \cdots \hat{x}_{j n} \mapsto \hat{x}_{j 1}^{\phi} \cdots \hat{x}_{j n}^{\phi}|0\rangle$. Thus (with $i:=\sqrt{-1}$ ) we get

$$
\begin{aligned}
\exp (i k \cdot x) * \exp (i q \cdot x) & =\exp \left(i K_{0}^{-1}(k) \cdot \hat{x}^{\phi}\right) \exp \left(i K_{0}^{-1}(q) \cdot \hat{x}^{\phi}\right)|0\rangle \\
& =\exp \left(i K_{0}^{-1}(k) \cdot \hat{x}^{\phi}\right)(\exp (i q \cdot x)) .
\end{aligned}
$$

If we define $\mathcal{D}(k, q)$ by

$$
\exp (i k \cdot x) * \exp (i q \cdot x)=\exp (i \mathcal{D}(k, q) x)
$$

then we obtain

$$
\mathcal{D}(k, q)=K\left(K_{0}^{-1}(k), q\right) .
$$

The linear dual $U(\mathfrak{g})^{*}$ of the enveloping algebra $U(\mathfrak{g})$, is a formal power series ring in the dual variable to the basis of $\mathfrak{g}$ [11, Section 10], with adic topology (of formal power series ring) and equipped with a topological coproduct, namely the transpose operator to the product [21]. If the dual is properly identified with the completed symmetric algebra of the dual to the Lie algebra $\hat{S}\left(\mathfrak{g}^{*}\right)$ with dual basis given by partial derivatives, then this coproduct may be described by several alternative means described in [18]. One of them is given by the rule $m_{*} \Delta(P)(f \otimes g)=P(f * g)$ where $P \in \hat{S}(\mathfrak{g})$ is viewed as a differential operator with constant coefficients evaluated at unity and $m_{*}(f \otimes g):=f * g$. Then

$$
\begin{aligned}
\partial^{j}\left(e^{i k x} * e^{i q x}\right) & =\partial^{j}\left(e^{i K_{0}^{-1}(k) \hat{x}^{\phi}}\left(e^{i q x}\right)\right)=i K_{j}\left(K_{0}^{-1}(k), q\right) e^{i K\left(K_{0}^{-1}(k), q\right) x} \\
& =i m_{*} K_{j}\left(K_{0}^{-1}(k \otimes 1), 1 \otimes q\right)\left(e^{i k x} \otimes e^{i q x}\right) \\
& =i m_{*} K_{j}\left(K_{0}^{-1}(-i \partial \otimes 1), 1 \otimes(-i \partial)\right)\left(e^{i k x} \otimes e^{i q x}\right) .
\end{aligned}
$$

Hence $\Delta\left(\partial^{j}\right)=i K_{j}\left(K_{0}^{-1}\left(-i \partial^{1} \otimes 1, \ldots,-i \partial^{n} \otimes 1\right),-i 1 \otimes \partial^{1}, \ldots,-i 1 \otimes \partial^{n}\right)$.

Heuristics. While the existence of $K_{l}$ satisfying (1) is discussed in Section 2 in greater generality, in our case there is a simple heuristics (pointed to the second author by S. Skryabin whom we thank): the exponentials in the appropriate completions of $U(\mathfrak{g})$ and $S(\mathfrak{g})$ should be group-like elements (as the exponentials of primitive elements always are) in the sense of Hopf algebras. The coalgebra isomorphism $\xi$ preserves the property of being group-like. We have just sketched the correspondence between the star product and the expressions like the left-hand side of (1). The product of group-like elements is group-like in a Hopf algebra; all group-likes in $U(\mathfrak{g})$ are exponentials (in the nilpotent Lie algebra case; otherwise we would need more precise argument for taking care of the completions needed for the general case).

\section{Operating on exponentials}

This section is of a ring-theoretic nature, and deals with the facts that are more general than the study of the realizations of Lie algebra type noncommutativity in the remainder of the article. Given a differential operator or a formal power series $F=F(d / d x)$, we show the existence of $K=K(\lambda, k)$ such that

$$
\exp (\lambda x F(d / d x))(\exp (k x))=\exp (K(\lambda, k) x)
$$

as a special case of a general fact which we prove not only for the arbitrary formal power series $F(d / d x)$ including a multidimensional version, but even for an arbitrary derivation $D$ 
(replacing $d / d x$ ), or a commuting family of derivations, on a fixed commutative ring, which is not necessarily the polynomial or power series ring, and not necessarily in characteristic zero. Linearity of the argument of the left-most exponential in $x$ is, however, essential.

Basic case. Let $A_{1}$ be the first Weyl algebra (over the ring $\mathbf{Q}$ of rational numbers or a ring containing $\mathbf{Q})$ with generators $x, d / d x$, and $k, \lambda$ formal commuting variables; by $\hat{A}_{1}$ we will mean its completion by the degree of differential operator, hence allowing formal series in $d / d x$. Then, we will show (see Corollary 2) that for any $F=F(d / d x) \in \mathbf{Q}[[d / d x]]$, there is a unique $\alpha=\alpha(\lambda, d / d x) \in \mathbf{Q}[[\lambda, d / d x]]$ such that in $\hat{A}_{1}[[\lambda, k]]$

$$
\exp (\lambda x F)=\sum_{s=0}^{\infty} \frac{x^{s} \alpha^{s}}{s !}
$$

To justify (4), we will essentially use just the fact that commuting with $x$ is a derivation of some subalgebra containing $F$. Of course, $[\alpha(\lambda, d / d x), x] \neq 0$ in general and the ordering between $x$ and $\alpha$ in summands $x^{s} \alpha^{s}$ above is essential. The right-hand side may be viewed symbolically as a normally ordered exponential $: \exp (x \alpha):$ (where $x$ 's are always at the left, and $\alpha$ 's always at the right).

Regarding that $\lambda$ and $d / d x$ commute and $d / d x$ is not of finite order, we can make a substitution $d / d x \mapsto k$ and define $\alpha(\lambda, k)$ (of course the result does not define an endomorphism of $\left.\hat{A}_{1}[[\lambda, k]]\right)$; it is a power series with the same coefficients but different argument. We will show that

$$
\exp (\lambda x F(d / d x))(\exp (k x))=\exp ((\alpha(\lambda, k)+k) x)
$$

holds true in $\hat{A}_{1}[[\lambda, k]]$.

Similarly, in the case of several variables $x_{1}, \ldots, x_{n}$,

$$
\exp \left(\lambda \sum_{i=1}^{n} x_{i} F_{i}\left(d / d x_{1}, \ldots, d / d x_{n}\right)\right)=\sum_{s_{1}, \ldots, s_{n}}^{\infty} \frac{x_{1}^{s_{1}} \cdots x_{n}^{s_{n}} \alpha_{1}^{s_{1}} \cdots \alpha_{n}^{s_{n}}}{s_{1} ! \cdots s_{n} !}
$$

for unique functions $\alpha_{i} \in \hat{A}_{n}[[\lambda]]$ which, of course, depend on the commutators $\left[x_{i}, F_{j}\right] \in \hat{A}_{n}$.

To investigate these questions we will work in an abstract algebra $\mathcal{S}^{\prime}$ which generalizes the Weyl algebra (possibly completed, with additional parameters). By a Q-algebra we mean an associative unital algebra over rationals.

Proposition 1. Let $\mathcal{S}^{\prime}$ be a $\mathbf{Q}$-algebra, and $\mathcal{S} \subset \mathcal{S}^{\prime}$ a commutative subalgebra. Let $x \in \mathcal{S}^{\prime}$ and $F \in S$ be such that the commutator $[, x]$ is a derivation of $\mathcal{S}$. Then (4) is true in $\mathcal{S}[[\lambda]]$ for $\alpha=\sum_{l=0}^{\infty} \lambda^{l} A_{1, l} / l !$ (where $A_{1, l} \in \mathcal{S}[[\lambda]]$ will be obtained below).

As $\alpha$ will be explicitly constructed one evidently has the existence of $K(\lambda, k)=\alpha(\lambda, k)+k$ which we seek above. Now, instead of commutator $[-, x]$ we consider an arbitrary derivation $D$ of $\mathcal{S}$ and we generalize the setup.

Notation. Let $\mathcal{S}$ be any commutative ring (not necessarily containing the rationals), $F \in \mathcal{S}$ an element and $D: \mathcal{S} \rightarrow \mathcal{S}$ a derivation. Define a double sequence $\left\{A_{s, l}\right\}_{s, l \geq 0}$ of elements in $\mathcal{S}$ as follows: $A_{s, l}=0$ unless $0 \leq s \leq l ; A_{0,0}=1$, and recursively $A_{s, l+1}=F \cdot\left(D\left(A_{s, l}\right)+A_{s-1, l}\right)$ for $l>1$.

Special values of $A_{r, s}$. In particular, $A_{0, l}=0$ for $l>0 ; A_{1, l+1}=F D\left(A_{1, l}\right)=(F D)^{l}(F)$ for $l \geq 0$ and $A_{s, s}=F^{s}$ for every $s \geq 0$.

The evaluation of a fixed derivation $D$ of a ring $S$ can be represented as a commutator with an element $T$ in an associated extension of the original ring, the Ore extension. Its underlying left $\mathcal{S}$-module is the free module of infinite rank underlying the ring of polynomials in one 
indeterminate $\mathcal{S}[T]$, but the multiplication is changed to the unique choice which is making it a ring, extends $\mathcal{S} \hookrightarrow \mathcal{S}[T]$, and satisfies

$$
T \cdot r=r T+D(r), \quad \forall r \in \mathcal{S}
$$

In particular, the Weyl algebras can be obtained as iterated Ore extensions of polynomial rings.

Theorem 1. For any $\mathcal{S}, \mathcal{S}^{\prime}, F, D$ as above and $s \geq 2$, the double sequence $A_{n, l}$ satisfies the integral recursion

$$
s A_{s, l}=\sum_{r=1}^{l+1-s}\left(\begin{array}{l}
l \\
r
\end{array}\right) A_{1, r} A_{s-1, l-r} .
$$

The upper limit of the sum on the right-hand side can harmlessly be extended up to $l-1$ : the additionally included summands anyway vanish. If we were in characteristics zero we could instead write the recursion for the $\tilde{A}_{s, l}=s ! A_{s, l} / l$ ! which would be a recursion of convolution type.

Proof of Theorem 1. The proof is by induction on $l$ : if $s>l$ the equation reads $0=0$, for $s=l$ it reads $s A_{s, s}=s A_{s-1, s-1} F$, because $A_{s, s}=F^{s}$; we just need to verify the step of induction from $(s, l)$ with $l \geq s$ to $(s, l+1)$. For this we write $A_{s, l+1}=F D\left(A_{s, l}\right)+A_{1,1} A_{s-1, l}$, substitute (6) for $A_{s, l}$ and apply $D$ using the Leibniz rule in each summand to obtain

$$
s A_{s, l+1}=s A_{1,1} A_{s-1, l}+\sum_{r=1}^{l-1}\left(\begin{array}{l}
l \\
r
\end{array}\right) F D\left(A_{1, r}\right) A_{s-1, l-r}+\sum_{r=1}^{l-1}\left(\begin{array}{l}
l \\
r
\end{array}\right) A_{1, r} F D\left(A_{s-1, l-r}\right) .
$$

Now $F D\left(A_{1, r}\right)=A_{1, r+1}$ and

$$
F D\left(A_{s-1, l-r}\right)=A_{s-1, l-r+1}-A_{1,1} A_{s-2, l-r},
$$

where the second summand on the right vanishes if $s=2$. Now we finish separately the case of $s=2$ and $s>2$.

For $s=2$ we obtain

$$
2 A_{2, l+1}=2 A_{1,1} A_{1, l}+\sum_{r=1}^{l-1}\left(\begin{array}{l}
l \\
r
\end{array}\right) A_{1, r+1} A_{1, l-r}+\sum_{r=1}^{l-1}\left(\begin{array}{l}
l \\
r
\end{array}\right) A_{1, r} A_{1, l-r+1} .
$$

After absorbing $A_{1,1} A_{1, l}$ into first sum as the additional $r=0$ summand and into the second sum as $r=l$ summand, and adding the two sums we obtain the required form.

For $s>2$ there are several differences. First of all $s A_{1,1} A_{s-1, l}$ should be split into $A_{1,1} A_{s-1, l}$ which is absorbed into the first sum as before, and $(s-1) A_{1,1} A_{s-1, l}$ which exactly cancels the additional sum coming from summands coming from additional $A_{1,1} A_{s-2, l-r}$ in (7). The third difference is that $A_{s-1, l} A_{1,1}$ which was absorbed to extend the upper limit in the second sum for $s=2$ does not need to be added for $s>2$ because the top limit of $l-1$ is anyway beyond the limit of vanishing terms.

Corollary 1. Let $k \geq 2$ and $2 \leq s=s_{1}+\cdots+s_{k}$ with $s_{i} \geq 1$. Then

$$
\frac{s !}{s_{1} ! \cdots s_{k} !} A_{s, l}=\sum_{l_{1}+\cdots+l_{k}=l, l>l_{i} \geq 1} \frac{l !}{l_{1} ! \cdots l_{k} !} A_{s_{1}, l_{1}} \cdots A_{s_{k}, l_{k}} .
$$


Proof. We first prove it for $k=2$. In that case, for $s_{1}=1$ this is the statement of the theorem above. Suppose now we have proven the statement for $s_{1} \geq p$. Then express $s_{2}=1+\left(s_{2}-1\right)$ and decompose $A_{s_{2}, l_{2}}$ into the sum of products of the form $A_{1, l_{2}^{\prime}} A_{s_{3}, l_{3}^{\prime}}$, and resum $A_{s_{1}, l_{1}}$ and $A_{1, l_{2}^{\prime}}$ coming from the first factor in the second sum. The coefficients can be easily compared.

For $k>2$ this is an easy induction on $k$ using the result for $k=2$ both for the basis and for the step of induction.

Suppose now $\mathcal{S}$ is a $\mathbf{Q}$-algebra and $D$ is $\mathbf{Q}$-linear derivation given by the commutator $[-, x]$ with a fixed element $x \in \mathcal{S}^{\prime}$ where $\mathcal{S}^{\prime} \supset \mathcal{S}$ is a Q-algebra containing $\mathcal{S}$. Let $\lambda$ be a formal variable. Then in $\mathcal{S}^{\prime}[[\lambda]]$

\section{Corollary 2.}

$$
\exp (\lambda x F)=\sum_{s=0}^{\infty} \frac{x^{s} \alpha^{s}}{s !}
$$

where $\alpha=\sum_{l=1}^{\infty} \lambda^{l} A_{1, l} / l$ ! and, of course, the commutator $[\alpha, x] \neq 0$ in general.

Proof. If we set $(x F)^{k}=\sum_{s=1}^{k} x^{s} B_{s, k-s}$ then we see that $B_{s, k-s}$ satisfy the recursion and initial conditions for $A_{s, k-s}$ above. Indeed, $\sum_{s=1}^{k} x^{s} B_{s, k-s} x F=\sum_{s=1}^{k} x^{s+1} B_{s, k-s} F+x^{s}\left(D B_{s, k-s}\right) F$ and we get the recursion after renaming the labels.

Thus the corollary follows from Corollary 1.

\section{Example 1.}

$$
\begin{aligned}
x\left(\frac{d}{d x}\right)^{l} \frac{x^{m+j(l-1)}}{(m+j(l-1)) !}= & (m+j(l-1))(m+j(l-1)-1) \cdots \\
& \times(m+j(l-1)-(l-1)) \frac{x^{m+(j-1)(l-1)}}{(m+j(l-1)) !} \\
= & (m+(j-1)(l-1)) \frac{x^{m+(j-1)(l-1)}}{(m+(j-1)(l-1)) !} .
\end{aligned}
$$

Therefore

$$
\frac{1}{j !}\left(x \frac{d^{l}}{d x^{l}}\right)^{j} \frac{x^{m+j(l-1)}}{(m+j(l-1)) !}=\frac{1}{j !} m(m+l-1) \cdots(m+(j-1)(l-1)) \frac{x^{m}}{m !} .
$$

Now $\left(x \frac{d^{l}}{d x^{l}}\right)^{j} x^{n}=0$ if $m:=n-(l-1) j<0$. Therefore

$$
e^{x \frac{d^{l}}{d x^{l}}} e^{k x}=\sum_{j=0}^{\infty} \sum_{m=0}^{\infty} \frac{\left(x(d / d x)^{l}\right)^{j}}{j !} \frac{x^{m+j(l-1)}}{(m+j(l-1)) !} k^{m+j(l-1)} .
$$

By the binomial formula

$$
\left(1-(l-1) k^{l-1}\right)^{\frac{-m}{l-1}}=\sum_{j=0}^{\infty} \frac{1}{j !} m(m-l+1) \cdots(m-(j-1)(l-1)) k^{j(l-1)} .
$$

Hence

$$
\left(\frac{k}{\left(1-(l-1) k^{l-1}\right)^{\frac{1}{l-1}}}\right)^{m}=\sum_{j=0}^{\infty} m(m-l+1) \cdots(m-(j-1)(l-1)) \frac{k^{m+j(l-1)}}{j !} .
$$


Therefore

$$
e^{x \frac{d^{l}}{d x^{l}}} e^{k x}=\sum_{m=0}^{\infty}\left(\frac{k}{\left(1-(l-1) k^{l-1}\right)^{\frac{1}{l-1}}}\right)^{m} \frac{x^{m}}{m !}=\exp \left(\frac{k x}{\left(1-(l-1) k^{l-1}\right)^{\frac{1}{l-1}}}\right)
$$

for $l=0,1,2, \ldots$ Therefore, for $\lambda=1, F=(d / d x)^{l}$, we have

$$
\alpha(\lambda, k)+k=K(\lambda, k)=\frac{k}{\left(1-(l-1) k^{l-1}\right)^{\frac{1}{l-1}}} .
$$

After this work appeared at arXiv, preprint [8] also appeared, where a formula equivalent to (8) was derived as a special case of a combinatorial method (see Fig. 1 and Chapter 6 in [8]).

It is easy to generalize our results to treat also the multivariable case (5) via ansatz

$$
\alpha_{i}=\sum_{l=0}^{\infty} \frac{\lambda^{l}}{l !} A_{0, \ldots, 1, \ldots, 0, l}
$$

where 1 is at $i$-th place. This time we study a commutative algebra $\mathcal{S}$ with $n$ commuting derivations $D_{i}$. The characteristics free recursion is this time for the $(n+1)$-tuple sequence of elements $A_{s_{1}, \ldots, s_{n}, l} \in \mathcal{S}[[\lambda]]$ :

$$
A_{s_{1}, \ldots, s_{n}, l+1}=\sum_{i=1}^{n} F_{i} \cdot\left(D_{i}\left(A_{s_{1}, \ldots, s_{n}, l}\right)+A_{s_{1}, \ldots, s_{i-1},\left(s_{i}\right)-1, s_{i+1}, \ldots, s_{i}, l}\right)
$$

with initial conditions $A_{0, \ldots, 0,0}=1$ and $A_{s_{1}, \ldots, s_{l}, 0}=0$ when at least one of the $s_{i} \neq 0$. Then it follows by a straightforward generalization of the proof in the case of one derivation that for all $s_{i j}$ where $1 \leq i \leq n, 1 \leq j \leq k$ and $s_{i}=\sum_{j=1}^{k} s_{i j}$,

$$
\frac{s_{1} ! \cdots s_{k} !}{s_{11} ! s_{12} ! \cdots s_{n k} !} A_{s_{1}, \ldots, s_{n}, l}=\sum_{l_{1}+\cdots+l_{k}=l \geq l_{i} \geq 1} \frac{l !}{l_{1} ! \cdots l_{k} !} A_{s_{11}, \ldots, s_{1 n}, l_{1}} \cdots A_{s_{k 1}, \ldots, s_{k n}, l_{k}} .
$$

\section{Formal differential equations}

We shall now exhibit some practical methods of calculating $K(\lambda, q)$ determined by

$$
\exp (\lambda x F(d / d x))(\exp (i q x))=\exp (K(\lambda, q) x)
$$

for formal parameter $\lambda$, real arguments $q$ and $x$, and formal series $F$.

In multivariate case, given $F(\partial)=F\left(\partial_{1}, \ldots, \partial_{n}\right)$ let

$$
K=K(\lambda, q)=\left(K_{1}(\lambda, q), \ldots, K(\lambda, q)\right)=\left(K_{1}\left(\lambda, q_{1}, \ldots, q_{n}\right), \ldots, K_{n}\left(\lambda, q_{1}, \ldots, q_{n}\right)\right)
$$

be defined by

$$
e^{K(\lambda, q) \cdot x}:=e^{\lambda x \cdot F(\partial)}\left(e^{q \cdot x}\right) .
$$

Then

$$
x \cdot \frac{\partial K}{\partial \lambda}(\lambda, q) e^{K(\lambda, q) x}=\frac{\partial}{\partial \lambda}\left(e^{\lambda x \cdot F(\partial)} e^{q \cdot x}\right)=x \cdot F(\partial) e^{\lambda x \cdot F(\partial)} e^{q \cdot x} .
$$

The right-hand side can by definition (9) written as

$$
x \cdot F(\partial) e^{\lambda x \cdot F(\partial)} e^{q \cdot x}=x \cdot F(K) e^{K(\lambda, q) \cdot x},
$$


but also as

$$
\begin{aligned}
e^{\lambda x \cdot F(\partial)} x \cdot F(\partial) e^{q \cdot x} & =e^{\lambda x \cdot F(\partial)} x \cdot F(q) e^{q \cdot x}=\sum_{i=1}^{n} F_{i}(q) e^{\lambda x \cdot F(\partial)} x_{i} e^{q x} \\
& =\sum_{i=1}^{n} F_{i}(q) \frac{\partial}{\partial q_{i}}\left(e^{\lambda x \cdot F(\partial)} e^{q \cdot x}\right)=\sum_{i=1}^{n} F_{i}(q) \frac{\partial}{\partial q_{i}}\left(e^{K(\lambda, q) \cdot x}\right) \\
& =\sum_{i, j} F_{i}(q) \frac{\partial K_{j}}{\partial q_{i}}(\lambda, q) x_{j} e^{K(\lambda, q) \cdot x} .
\end{aligned}
$$

Thus we obtain

$$
\sum_{j} x_{j} F_{j}(K) e^{K(\lambda, q) \cdot x}=\sum_{i, j} F_{i}(q) \frac{\partial K_{j}}{\partial q_{i}}(\lambda, q) x_{j} e^{K(\lambda, q) \cdot x} .
$$

After multiplying by $\exp (-K(\lambda, q) \cdot x)$ both sides we get expressions linear in $x_{j}$. Therefore, equating the coefficients of $x_{1}, \ldots, x_{n}$, we obtain the system

$$
F_{j}(K(\lambda, q))=\sum_{i} F_{i}(q) \frac{\partial K_{j}}{\partial q_{i}}(\lambda, q)=\frac{\partial K_{j}}{\partial \lambda}(\lambda, q),
$$

where $j=1, \ldots, n$ and the boundary condition is $K(0, q)=q$.

Let $n=1$ and $F=(d / d x)^{l}, l>0$. Then the equations become

$$
K^{l}=q^{l} \frac{\partial K}{\partial q}=\frac{\partial K}{\partial \lambda}, \quad K=K(\lambda, q), \quad K(0, q)=q
$$

By integrating $K^{l}=\partial K / \partial \lambda$ we obtain that $K^{-l+1}=(1-l)(\lambda+C(q))$ where $C=C(q)$ is some function of $q$. Thus

$$
\frac{\partial K^{1-l}}{\partial q}=(1-l) \frac{d C}{d q}
$$

where the left-hand side evaluates to $(1-l) K^{-l} \frac{\partial K}{\partial q}=(1-l) K^{-l} K^{l} / q^{l}=(1-l) q^{-l}$. Therefore $C(q)=q^{1-l} /(1-l)+C_{0}$ and it is easy to see that $C_{0}=0$. Therefore $K^{1-l}=\lambda(1-l)+q^{1-l}$, hence, for $l>0$,

$$
e^{\lambda x \frac{d^{l}}{d x^{l}}} e^{q x}=\exp \left(\left(q^{1-l}+\lambda(1-l)\right)^{\frac{1}{1-l}} q x\right)=\exp \left(\frac{q x}{\left(1-\lambda(l-1) q^{l-1}\right)^{1 /(l-1)}}\right),
$$

in agreement with the direct summation in Example 1 (for $\lambda=1$ ).

A formal solution. For a parameter $\mu$, and $1 \leq i \leq n$, define operator $Q_{i}(\mu)$ by

$$
Q_{i}(\mu)=e^{-\mu x \cdot F(\partial)} \partial_{i} e^{\mu x \cdot F(\partial)}=\sum_{n=0}^{\infty} \frac{\mu^{n} \operatorname{ad}^{n}(-x \cdot F(\partial))}{n !}\left(\partial_{i}\right) .
$$

Now for any $R=R(\partial)$, notice

$$
\left[-x_{j} F_{j}(\partial), R\right]=F_{j} \frac{\partial}{\partial\left(\partial_{j}\right)} R=: F_{j} \delta_{j} R
$$

because $\left[F_{j}, R\right]=0$. Then

$$
\operatorname{ad}^{n}(-x \cdot F(\partial)) F_{i}=-\sum_{j_{1}, \ldots, j_{n}} F_{j_{1}} \delta_{j_{1}}\left(F_{j_{2}} \delta_{j_{2}}\left(\ldots\left(F_{j_{n}} \delta_{j_{n}}\left(F_{i}\right)\right) \ldots\right)\right) .
$$


Thus we obtain a formal solution

$$
Q_{i}(\mu)=\partial_{i}+\frac{\exp (\mu \mathcal{O})-1}{\mathcal{O}} F_{i}(\partial)
$$

where $\mathcal{O}=\mathcal{O}(\partial)=\sum_{i} F_{i}(\partial) \delta_{i}$. Clearly

$$
Q_{i}(\mu) e^{q \cdot x}=e^{-\mu x \cdot F} \partial_{i} e^{\mu x \cdot F} e^{q \cdot x}=e^{-\mu x \cdot F} K_{i}(\mu, q) e^{K(\mu, q) \cdot x}=K_{i}(\mu, q) .
$$

Therefore

$$
K_{i}(\mu, q)=q_{i}+\frac{\exp (\mu \mathcal{O}(q))-1}{\mathcal{O}(q)} F_{i}(q)
$$

For us the most important case will be $F_{i}(\partial)=\sum_{j} k_{j} \phi_{j i}(\partial)$ where

$$
\sum_{i} x_{i} F_{i}(\partial)=\sum_{i j} k_{j} x_{i} \phi_{i j}(\partial)=\sum_{j} k_{j} \hat{x}_{j}^{\phi}
$$

for $\hat{x}_{j}^{\phi}:=\sum_{i} x_{i} \phi_{i j}(\partial)$.

The formal solution can alternatively be obtained using the expressions $A_{r, s}$ in the recursion from Section 2. Indeed, $K(\mu, q)=\alpha(\mu, q)+q$. For simplicity, we will write it out in one variable. By Corollary 2, in the notation used there, $\alpha=\sum_{l=1}^{\infty} \mu^{l} A_{1, l} / l$ !, and the recursion gives the special values $A_{1, l}=(F D)^{l-1} F$, for $l \geq 1$. Thus we obtain

$$
K(\mu, q)=q+\sum_{l=1}^{\infty} \mu^{l}(F D)^{l-1} F / l !=q+\frac{\exp (\mu F D)-1}{F D} F .
$$

\section{Examples related to $s u(2)$}

We are now going to consider two different realizations of $s u(2)$. We will slightly modify the problem: the variable $\lambda$ will be replaced by three parameters forming a vector $\vec{P}_{1}$ with length $P_{1}$. General vector $q$ from above will be denoted $\vec{P}_{2}$. Thus instead of $K(\lambda, q)$ we want to find (for some realization $\left.\phi=\left(\phi_{b}^{a}\right)\right)$ the function $K=K\left(\vec{P}_{1}, \vec{P}_{2}\right)=K_{\phi}\left(\vec{P}_{1}, \vec{P}_{2}\right)$ in the exponent. Tricks with vector calculus and geometrically well-chosen substitutions are useful in finding the solutions. The differential equations will not be directly modified from the previous section, but rather rederived on the spot in a way introducing some useful auxiliary variables. Compare that the formal solution from the previous section are obtained using essentially the same variables (up to imaginary unit).

Below we shall use a basis $\hat{x}_{1}, \hat{x}_{2}, \hat{x}_{3}$ of $s u(2)$ satisfying $\left[\hat{x}_{a}, \hat{x}_{b}\right]=i \kappa \epsilon_{a b c} \hat{x}_{c}$, where $\kappa$ is a small parameter (this strange convention is an adaptation for the applications to modeling some noncommutative deformations of a space-time). Define the auxiliary variables

$$
\hat{P}_{a}(\mu):=e^{-i \mu k \cdot \hat{x}} \hat{P}_{a}(0) e^{+i \mu k \cdot \hat{x}},
$$

where $\hat{P}_{a}(0)=\hat{p}_{a}=-i \partial_{a}$. Thus

$$
\frac{d \hat{P}_{a}}{d \mu}(\mu)=e^{-i \mu k \cdot \hat{x}}\left[-i k \cdot \hat{x}, \hat{P}_{a}(0)\right] e^{+i \mu k \cdot \hat{x}} .
$$

The realization of $U(s u(2))$ of Freidel and Livine [12]. This realization is also used in [13] in the context of study of a noncommutative Fourier transform used to relate a group field theory related to a spin-foam model motivated by $3 d$ quantum gravity to a noncommutative field theory. 
In our language their star product is coming from a realization via formal differential operators of infinite order, is (with Einstein summation convention) given by

$$
\hat{x}_{a}^{\phi}=x_{b} \phi_{b a}=x_{a} \sqrt{1+\kappa^{2} \partial^{2}}+i \epsilon_{a b c} \kappa x_{b} \partial_{c}, \quad \phi_{b a}=\delta_{b a} \sqrt{1+\kappa^{2} \partial^{2}}+i \kappa \epsilon_{a b c} \partial_{c} .
$$

Elements of $U(s u(2))$ in this realization in the semicompleted Weyl algebra act as formal differential operators on its standard module - the Fock space which is the symmetric algebra $S(s u(2))$ with unit playing the role of Fock vacuum $1=\exp (i 0 \cdot x)=:|0\rangle$. We rescale all by imaginary units to define $K$ by $\exp \left(i k \cdot \hat{x}^{\phi}\right) \exp (i q \cdot x)=\exp (i K(k, q) \cdot x)$. The action in the realization is $\exp \left(i k \cdot \hat{x}^{\phi}\right)|0\rangle=\exp (i K(k, 0) \cdot \hat{x})$.

We will use the notation and the relation between $K, K_{0}$ and the coproduct from Section 1. For $\hat{p}_{a}=-i \partial_{a}$ we have

$$
\left[\hat{x}_{a}, \hat{p}_{b}\right]=i \sqrt{1-\kappa^{2} \hat{p}^{2}} \delta_{a b}-i \kappa \epsilon_{a b c} \hat{p}_{c}, \quad \hat{P}_{a}(0)=-i \partial_{a} .
$$

Then $\left[\hat{x}_{a}, \partial_{b}\right]=\phi_{a b}$, what implies

$$
\frac{d \hat{P}_{a}}{d \mu}=k_{a} \sqrt{1-\kappa^{2} \hat{P}^{2}}+\epsilon_{a b c} k_{b} \hat{P}_{c}
$$

In these formulas the operations involving $\partial$ are understood as acting on linear combinations of Fourier components $\exp (i q \cdot \vec{x})$, which are the eigenvectors, with values of $-i \partial_{a}$ equal to $q_{a}$. From now on we fix a single Fourier component $\exp (i q \cdot \vec{x})$ and write equations for $P$ which is the corresponding eigenvalue of $\hat{P}$.

In solving the equations it is useful to utilize full vector notation, hence writing $\vec{k}, \vec{q}$. We also make shortcuts

$$
L:=\vec{k} \cdot \vec{P}, \quad P^{2}:=\sum_{a}\left(P_{a}\right)^{2}, \quad k^{2}=|\vec{k}|^{2}, \quad q^{2}=|\vec{q}|^{2} .
$$

Then

$$
\begin{aligned}
& \frac{d L}{d \mu}=k^{2} \sqrt{1-\kappa^{2} P^{2}}, \quad \frac{1}{2} \frac{d P^{2}}{d \mu}=L \sqrt{1-\kappa^{2} P^{2}}, \\
& \frac{d}{d \mu} \sqrt{1-\kappa^{2} P^{2}}=-\kappa^{2} \frac{d P^{2} / d \mu}{2 \sqrt{1-\kappa^{2} P^{2}}}=-\kappa^{2} L .
\end{aligned}
$$

Now we derive one more time,

$$
-\frac{1}{\kappa^{2}} \frac{d^{2}}{d \mu^{2}} \sqrt{1-\kappa^{2} P^{2}}=\frac{d L}{d \mu}=k^{2} \sqrt{1-\kappa^{2} P^{2}} .
$$

We seek the solution of that differential equation for $\sqrt{1-\kappa^{2} P^{2}}$ in the form

$$
\sqrt{1-\kappa^{2} P^{2}}=c_{1} \cos \kappa|\vec{k}| \mu+c_{2} \sin \kappa|\vec{k}| \mu .
$$

Then of course $L=\frac{|\vec{k}|}{\kappa}\left(c_{1} \cos \kappa|\vec{k}| \mu+c_{2} \sin \kappa k \mu\right)$, and $P(\mu=0)=q$, hence $c_{1}=\sqrt{1-\kappa^{2} q^{2}}$. On the other hand, $L(\mu=0)=\vec{q} \cdot \vec{k}$, thus $L(\mu=0)=|\vec{k}| \frac{c_{2}}{\kappa}=\vec{q} \cdot \vec{k}$, hence $c_{2}=-\frac{\vec{q} \cdot \vec{k}}{|\vec{k}|} \kappa$. Thus

$$
L=\frac{|\vec{k}|}{\kappa} \sqrt{1-\kappa^{2} q^{2}} \sin (\kappa|\vec{k}| \mu)+\vec{q} \cdot \vec{k} \cos (\kappa|\vec{k}| \mu)
$$

We seek for solution for $\vec{P}$ in the form $\vec{P}=f_{1} \vec{k}+f_{2} \vec{q}+f_{3} \vec{k} \times \vec{q}$. The equation

$$
\frac{d \vec{P}}{d \mu}+\vec{k} \sqrt{1-\kappa^{2} P^{2}}+\kappa \vec{k} \times \vec{P}
$$


becomes in these terms

$$
\frac{d f_{1}}{d \mu} \vec{k}+\frac{d f_{2}}{d \mu} \vec{q}+\frac{d f_{3}}{d \mu} \vec{k} \times \vec{q}=\sqrt{1-\kappa^{2} P^{2}} \vec{k}+\kappa f_{2} \vec{k} \times \vec{q}+\kappa f_{3}\left((\vec{q} \cdot \vec{k}) \vec{k}-k^{2} \vec{q}\right),
$$

what amounts to the system

$$
\frac{d f_{1}}{d \mu}=\vec{k} \sqrt{1-\kappa^{2} P^{2}}+\kappa \vec{q} \cdot \vec{k} f_{3}, \quad \frac{d f_{2}}{d \mu}=-\kappa \vec{q} \cdot \vec{k} f_{3}, \quad \frac{d f_{3}}{d \mu}=\kappa f_{2} .
$$

The latter two give

$$
\frac{d f_{2}}{d \mu}=-\kappa^{2} k^{2} f_{2}
$$

hence

$$
f_{2}=d_{1} \cos (\kappa|\vec{k}| \mu)+d_{2} \sin (\kappa|\vec{k}| \mu), \quad f_{3}=\frac{d_{1}}{k} \sin (\kappa|\vec{k}| \mu)-\frac{d_{2}}{k} \cos (\kappa|\vec{k}| \mu) .
$$

The boundary conditions are $f_{2}(0)=1, f_{3}(0)=0$, hence $d_{2}=0, d_{1}=1$

$$
\vec{P}=f_{1} \vec{k}+\cos (\kappa|\vec{k}| \mu) \vec{q}+\frac{1}{|\vec{k}|} \sin (\kappa|\vec{k}| \mu) \vec{k} \times \vec{q} .
$$

Forming the inner product of this equation with $\vec{k}$ and recalling the value of $L$ we get the condition (both sides are equal to $L$ )

$$
\begin{aligned}
& k^{2} f_{1}+\vec{q} \cdot \vec{k} \cos (\kappa|\vec{k}| \mu)=\frac{k}{\kappa} \sqrt{1-\kappa^{2} q^{2}} \sin (\kappa|\vec{k}| \mu)+\vec{q} \cdot \vec{k} \cos (\kappa|\vec{k}| \mu), \\
& \vec{P}=\frac{|\vec{k}|}{\kappa k} \sqrt{1-\kappa^{2} q^{2}} \sin (\kappa|\vec{k}| \mu)+\vec{q} \cos (\kappa|\vec{k}| \mu)+\frac{1}{|\vec{k}|} \vec{k} \times \vec{q} \sin (\kappa|\vec{k}| \mu) .
\end{aligned}
$$

Of course, then $K(\vec{k}, \vec{q})=\vec{P}(\mu=1)$ and $\overrightarrow{\mathcal{D}}(\vec{k}, \vec{q})$ is then evaluated by (3) to obtain

$$
\overrightarrow{\mathcal{D}}(\vec{k}, \vec{q})=\sqrt{1-\kappa^{2} k^{2}} \vec{k}+\sqrt{1-\kappa^{2} k^{2}} \vec{q}-\kappa \vec{k} \times \vec{q} .
$$

The symmetric realization or ordering is defined via the condition $e^{i \sum_{\alpha} k_{\alpha} \hat{x}_{\alpha}^{\phi}}|0\rangle=e^{i \sum_{\alpha} k_{\alpha} x_{\alpha}}$. In other words, $K_{0}$ from (2) is the identity. The composition of the realization $\hat{x} \mapsto \hat{x}^{\phi}$ and the projection on the vacuum in Fock space is then the inverse of the symmetrization map [11]. We will now study $s u(2)$ in this realization.

For $s u(2)$ we shall now use the basis proportional to $\sigma$-matrices $\hat{x}_{i}=\frac{1}{2} \sigma_{i}$; that basis satisfies $\left[\hat{x}_{i}, \hat{x}_{j}\right]=i \epsilon_{i j k} \hat{x}_{k}$, what follows from a useful identity $\sigma_{i} \sigma_{j}=\delta_{i j} \mathbf{1}+i \epsilon_{i j k} \sigma_{k}$. Then

$$
e^{i k \hat{x}}=e^{i \vec{k} \vec{\sigma}}=\sum_{n=0}^{\infty} \frac{1}{n !}\left(i \vec{k} \frac{\vec{\sigma}}{2}\right)^{n}=\cos |k|+i(\vec{k} \vec{\sigma}) \sin |k| .
$$

In the symmetric ordering, the vector function $\overrightarrow{\mathcal{D}}(\vec{k}, \vec{q})$ from formula (3) is determined by

$$
e^{i \vec{q} \vec{x}} * e^{i \vec{k} \vec{x}}=e^{i \overrightarrow{\mathcal{D}}(\vec{k}, \vec{q}) \vec{x}}=\cos |\overrightarrow{\mathcal{D}}|+\frac{i \overrightarrow{\mathcal{D}} \vec{x}}{|\overrightarrow{\mathcal{D}}|} \sin |\overrightarrow{\mathcal{D}}| .
$$

We need to multiply the expression in the left hand side and we easily get

$$
\begin{aligned}
& \cos |\overrightarrow{\mathcal{D}}|=\cos |\vec{k}| \cos |\vec{q}|-\frac{\vec{k} \vec{q}}{|\vec{k}||\vec{q}|} \sin |\vec{k}| \sin |\vec{q}|, \\
& \frac{\overrightarrow{\mathcal{D}}}{|\overrightarrow{\mathcal{D}}|} \sin |\overrightarrow{\mathcal{D}}|=\frac{\vec{k}}{|\vec{k}|} \sin |\vec{k}| \cos |\vec{q}|+\frac{\vec{q}}{|\vec{q}|} \cos |\vec{k}| \sin |\vec{q}|-\frac{\vec{k} \times \vec{q}}{|\vec{k}||\vec{q}|} \sin |\vec{k}| \sin |\vec{q}| .
\end{aligned}
$$


This corresponds to the realization

$$
\hat{x}_{i}=x_{i}+\frac{1}{2} \epsilon_{i j k} x_{j} p_{k}+\left(x_{i}-\frac{\vec{x} \vec{p}}{p^{2}} p_{i}\right)\left(\frac{p}{2} \operatorname{coth} \frac{p}{2}-1\right),
$$

where $p_{i} \rightarrow-i \partial_{i}$. This can be used to obtain $K$ as in the realizations above. The equation $\frac{d P_{i}}{d \mu}=\phi_{i j} k_{j}$ is then for Fourier component $\exp (i \vec{q} \cdot \vec{x})$

$$
\frac{d P_{i}}{d \mu}=k_{i}-\frac{1}{2} \epsilon_{i j k} k_{j} q_{k}+\left(k_{i}-\frac{k_{j} q_{j}}{q^{2}} q_{i}\right)\left(\frac{q}{2} \operatorname{coth} \frac{q}{2}-1\right) .
$$

One may solve the equations looking again the solution in the form $P(\mu)=P(\mu, \vec{k}, \vec{q})=g_{1} \vec{k}+$ $g_{2} \vec{q}+g_{3} \vec{k} \times \vec{q}$.

Setting $K(\vec{k}, \vec{q})=P(1, \vec{k}, \vec{q})$ one obtains $\mathcal{D}(\vec{k}, \vec{q})=K\left(K_{0}^{-1}(\vec{k}, \vec{q})\right)$ as before, with $K_{0}$ being the identity in the symmetric ordering, hence $\mathcal{D}=K$. This way $(\vec{k}, \vec{q}) \mapsto \mathcal{D}(\mu \vec{k}, \vec{q})$ satisfies the equation for $P=P(\mu, \vec{k}, \vec{q})$.

\section{Relation to works on star exponential}

In deformation quantization, the $n$-th Weyl algebra $A_{n}$ is often identified with a subalgebra of the Moyal algebra, i.e. $C^{\infty}(V)[[h]]$, where $V$ is the $2 n$-dimensional flat phase space with coordinates $x_{1}, \ldots, x_{n}, p_{1}, \ldots, p_{n}$ with the standard symplectic form, and the product involved is the Moyal star product $\star_{h}[3,6,7]$. Thus the realization for generators $\hat{x}^{\phi}$ can be considered as a function of the form $\sum_{i} x_{i} \phi_{j}^{i}(p)$ in the Moyal algebra. One wants to compute the action of $\exp \left(\sum_{j} k_{j} \hat{x}_{j}^{\phi}\right)$ on some $g(x)$, which is usually also an exponential. In the Moyal representation, one replaces $\hat{x}^{\phi}$ with a function of $x$ and $p$ as above and, in the power series expansion for the exponential, replaces the usual product by the star product. Differential equations and other techniques for computing such star exponentials are known (see e.g. [3]). Now we want to act on $g(x)$. For this one can express the exponential involving $\hat{x}^{\phi}$ as a star exponential for the Moyal star product, compute the star product with $g(x)$ and then act on the Fock space to project to a function of $x$ 's only. For this, the Moyal interpretation of Weyl algebra may be suboptimal, because the functions are in the symmetric Weyl ordering, while for the effective computing of the action on the Fock space (i.e. on the space of polynomials in $x_{1}, \ldots, x_{n}$ ) one usually needs a polarized form with derivatives pushed to the right hand side. The point of Section 2 is essentially a method of polarizing exponentials by a neat recursion. Our approach is instead to do the whole thing in a single step, either by recursion for coefficients in formal power series (Section 2), by a formal solution, or by solving a differential equation.

Our particular interest was in functions $\mathcal{D}(k, q)$ and $K(k, q)$ from Section 1 , where it is shown that $\mathcal{D}(k, q)$ is related to the coproduct for deformed momenta. This coproduct in the sense of Hopf algebras, is for this case, in noncommutative geometry interpreted as a deformed addition of momenta [14], which is neither studied, nor has much significance in the deformation quantization program. While in deformation quantization one quantizes the phase space, in our situation $[10,18,19,21]$ one just deforms the coordinate space (thus our star products will be just for functions of the form $f(x)$ and not $f(x, p)$ ) directly, while for finding the tangent and cotangent bundles, as well as for the deformations of the Poincaré algebra, one uses Hopf algebraic techniques, like deformed Leibniz rules [18], Heisenberg double [21] etc. Thus, we needed and computed very specific expressions, leading to the computation of function $\mathcal{D}(k, q)$, hence amounting to a new technique for computing the coproduct for deformed momenta (for a different approach see [10]).

Somewhat more generally, than in the rest of the article concerned with Weyl algebras, Section 2 is concerned with certain formal expressions involving a derivation on a general ring, 
and is partly beyond the scope of the usual Weyl algebra, but the rest is about calculations involving Weyl algebras. This is also beyond the case of Lie algebras, as such formal expressions do not necessarily close a Lie algebra.

Realization of Lie algebras by $\hat{x}_{j}{ }^{\phi}=\sum_{i} x_{i} \phi_{j}^{i}(\partial)$ can be obtained by interpreting Lie algebras as vector fields on the group and computing them in some coordinates around unit element (cf. [11, Sections 7-9]). More generally, one can find similar expressions from other actions on smooth manifolds. However, the actions do not need to exist beyond formal neighborhood in general, as we do not ask the formal power series for $\phi_{j}^{i}$ to have positive radius of convergence. Thus, in some cases, when the convergence (and smoothness) allows, we can consider our expressions as coming from a well known setup for quantization in the differential geometric setup. However, in full generality, the geometry of our paper is (like in [11, Sections 7-9]) concerned with vector fields on formal neighborhood of the unit of the Lie group.

\section{Conclusion and further questions}

We have exhibited several approaches to the exponential operators linear in variables and with arbitrary formal power series dependence in the partial derivatives, including direct summations, formal operator solutions and solving differential equations. We have shown much detail for the case of two realizations of $s u(2)$. These equations are specifically interesting for physical applications $[12,14,15,17]$ in the study of noncommutative spaces of Lie type via realizations by the differential operators of specific type.

While we defined the functions $K(k, q)$ and $\mathcal{D}(k, q)$ just formally in the relation to exponential expressions (cf. Section 1), computing them (up to some changes of variables) effectively computes also the addition of momenta on the noncommutative space, or equivalently, the coproduct on the space of dual variables $[14,18]$. This gives an important physical application of the method present here.

We remained within a formal approach (in the sense of formal power series). The analytic uniformization methods from [5] could also be used for similar study.

One can choose some reasonably big subspace of $\hat{S}(\mathfrak{g})$ to which the star product extends well, making it a topological algebra. Articles in deformation quantization studied such questions also in analytic setups. But even in the simple cases, e.g. when $\xi$ is the symmetrization map, defining a convenient subspace with well-defined star product and its topology is nontrivial.

The Raševskiî's associative hyper-envelope of a Lie algebra $\mathfrak{g}$ is a completion of $U(\mathfrak{g})$ by means of a countable family of norms $\hat{f} \mapsto\|\hat{f}\|_{\epsilon}$ for all $\epsilon$ in an arbitrary fixed family of positive numbers having 0 as an accumulation points, where

$$
\|\hat{f}\|_{\epsilon}=\max _{s_{1}, \ldots, s_{n}} \epsilon^{-\left(s_{1}+s_{2}+\cdots+s_{n}\right)}\left|f_{s_{1} \ldots s_{n}}\right|
$$

for $s_{1}+\cdots+s_{N}=s$, and where $f_{s_{1}, \ldots, s_{n}}$ is the Taylor coefficient in the front of $x_{1}^{s_{1}} \cdots x_{n}^{s_{n}}$ of the commutative polynomial $f=e^{-1}(\hat{f})$, where $e$ is the symmetrization map. Here $x_{1}, \ldots, x_{n}$ is any fixed basis of $\mathfrak{g}$, viewed as commutative coordinates. It is nontrivial and proved by Raševskii in [20] that the algebra multiplication in $U(\mathfrak{g})$ is continuous in this topology and hence that the completion of the $U(\mathfrak{g})$ as a countably normed vector space carries the unique structure of a topological algebra extending the algebra operations on $U(\mathfrak{g})$. It may be tried to use the same definition with $e$ replaced by another coalgebra isomorphism $\xi: S(\mathfrak{g}) \rightarrow U(\mathfrak{g})$. The second author (Z.S.) will show in a future publication that, under mild conditions on $\xi$, verifiable in many known examples, this modified definition results in a completion of $U(\mathfrak{g})$ isomorphic as a topological algebra. 


\section{Acknowledgements}

We thank the Croatia MSES projects for partial supports: 098-0000000-2865 (S.M. and Z.S.), 037-0372794-2807 (Z.Š.) and 037-0000000-2779 (D.S.).

\section{References}

[1] Amelino-Camelia G., Arzano M., Coproduct and star product in field theories on Lie-algebra noncommutative space-times, Phys. Rev. D 65 (2002), 084044, 8 pages, hep-th/0105120.

[2] Arnal D., Cortet J.C., *-products in the method of orbits for nilpotent groups, J. Geom. Phys. 2 (1985), 83-116.

[3] Arnal D., Cortet J.C., Molin P., Pinczon G., Covariance and geometrical invariance in * quantization, J. Math. Phys. 24 (1983), 276-283.

[4] Aschieri P., Lizzi F., Vitale P., Twisting all the way: from classical mechanics to quantum fields, Phys. Rev. D 77 (2008), 025037, 16 pages, arXiv:0708.3002.

[5] Barron K., Huang Y.Z., Lepowsky J., Factorization of formal exponentials and uniformization, J. Algebra 228 (2000), 551-579, math.QA/9908151.

[6] Bayen F., Flato M., Fronsdal C., Lichnerowicz A., Sternheimer D., Deformation theory and quantization. I. Deformations of symplectic structures, Ann. Physics 111 (1978), 61-110.

[7] Bayen F., Flato M., Fronsdal C., Lichnerowicz A., Sternheimer D., Deformation theory and quantization. II. Physical applications, Ann. Physics 111 (1978), 111-151.

[8] Blasiak P., Flajolet P., Combinatorial models of creation-annihilation, Ann. Physics 65 (2011), Art. B65c, 78 pages, arXiv:1010.0354.

[9] Borowiec A., Pachoł A., $\kappa$-Minkowski spacetimes and DSR algebras: fresh look and old problems, SIGMA 6 (2010), 086, 31 pages, arXiv:1005.4429.

[10] Dimitrijević M., Meyer F., Möller L., Wess J., Gauge theories on the $\kappa$-Minkowski spacetime, Eur. Phys. J. C Part. Fields 36 (2004), 117-126, hep-th/0310116.

[11] Durov N., Meljanac S., Samsarov A., Škoda Z., A universal formula for representing Lie algebra generators as formal power series with coefficients in the Weyl algebra, J. Algebra 309 (2007), 318-359, math.RT/0604096.

[12] Freidel L., Livine E.R., 3D quantum gravity and effective noncommutative quantum field theory, Phys. Rev. Lett. 96 (2006), 221301, 4 pages, hep-th/0512113.

[13] Freidel L., Majid S., Noncommutative harmonic analysis, sampling theory and the Duflo map in $2+1$ quantum gravity, Classical Quantum Gravity 25 (2008), 045006, 37 pages, hep-th/0512113.

[14] Halliday S., Szabo R.J., Noncommutative field theory on homogeneous gravitational waves, J. Phys. A: Math. Gen. 39 (2006), 5189-5225, hep-th/0602036.

[15] Kathotia V., Kontsevich's universal formula for deformation quantization and the Campbell-BakerHausdorff formula, Internat. J. Math. 11 (2000), 523-551, math.QA/9811174.

[16] Kontsevich M., Deformation quantization of Poisson manifolds, Lett. Math. Phys. 66 (2003), 157-216, q-alg/9709040.

[17] Meljanac S., Krešić-Jurić S., Stojić M., Covariant realizations of kappa-deformed space, Eur. Phys. J. C Part. Fields 51 (2007), 229-240, hep-th/0702215.

[18] Meljanac S., Škoda Z., Leibniz rules for enveloping algebras, arXiv:0711.0149, the latest version available at http://www.irb.hr/korisnici/zskoda/scopr5.pdf.

[19] Meljanac S., Stojić M., New realizations of Lie algebra kappa-deformed Euclidean space, Eur. Phys. J. C Part. Fields 47 (2006), 531-539, hep-th/0605133.

[20] Raševskiŭ P.K., Associative superenvelope of a Lie algebra and its regular representation and ideals, Trudy Moskov. Mat. Obšč 15 (1966), 3-54.

[21] Škoda Z., Heisenberg double versus deformed derivatives, Internat. J. Modern Phys. A 26 (2011), 4845-4854, arXiv:0909.3769.

[22] Škoda Z., Twisted exterior derivatives for enveloping algebras, arXiv:0806.0978. 